\title{
Unilateral fixation for treatment of occipitocervical instability in children with congenital vertebral anomalies of the craniocervical junction
}

\author{
Marcus D. Mazur, MD, Vijay M. Ravindra, MD, and Douglas L. Brockmeyer, MD \\ Department of Neurosurgery, Clinical Neurosciences Center, University of Utah, Salt Lake City, Utah
}

OBJECT Patients with occipitocervical (OC) instability from congenital vertebral anomalies (CVAs) of the craniocervical junction (CCJ) often have bony abnormalities that make instrumentation placement difficult. Within this patient population, some bilateral instrumentation constructs either fail or are not feasible, and a unilateral construct must be used. The authors describe the surgical management and outcomes of this disorder in patients in whom unilateral fixation constructs were used to treat $\mathrm{OC}$ instability.

METHODS From a database of $O C$ fusion procedures, the authors identified patients who underwent unilateral fixation for the management of $\mathrm{OC}$ instability. Patient characteristics, surgical details, and radiographic outcomes were reviewed. In each patient, CT scans were performed at least 4 months after surgery to evaluate for fusion.

RESULTS Eight patients with CVAs of the CCJ underwent unilateral fixation for the treatment of OC instability. For 4 patients, the procedure occurred after a bilateral $\mathrm{OC}$ construct failed or infection forced hardware removal. For the remainder, it was the primary procedure. Two patients required reoperation for hardware revision and 1 developed nonunion requiring revision of the bone graft. Ultimately, 7 patients demonstrated osseous fusion on CT scans and 1 had a stable fibrous union.

CONCLUSIONS These findings demonstrate that a unilateral $\mathrm{OC}$ fixation is effective for the treatment of $\mathrm{OC}$ instability in children with CVAs of the CCJ in whom bilateral screw placement fails or is not feasible.

http://thejns.org/doi/abs/10.3171/2015.1.FOCUS14787

KEY WORDS craniocervical; instability; occipitocervical fusion; pediatric; congenital vertebral anomalies; unilateral; spine; instrumentation

$\mathrm{T}$ HE surgical management of occipitocervical (OC) instability from congenital vertebral anomalies (CVAs) is challenging. Occipitocervical instability may result from a variety of bony or soft-tissue abnormalities, including but not limited to condylar dysplasia, C-1 hemi-rings, odontoid dysgenesis, and ligamentous laxity. $3,4,8,12,15$ Patients with these abnormalities often present early in life. In many cases, patients are asymptomatic, and the CVAs are diagnosed during surveillance imaging for congenital syndromes, such as spondyloepiphyseal dysplasia (SED) or Down syndrome. In other cases, patients are symptomatic from medullary or spinal cord compression and present with myelopathy and/or bulbar findings.
A standard biomechanical axiom states that to achieve the torsional rigidity necessary to facilitate fusion at the craniocervical junction (CCJ), it is necessary to have bilateral fixation. This concept has been passed down over many years, and has been examined in a small number of biomechanical studies that have evaluated atlantoaxial fixation. ${ }^{13,14}$ In certain circumstances, such as when bone is unilaterally destroyed by tumor or trauma or there are developmental skeletal abnormalities, however, bilateral OC fixation is either unsafe or impossible. A common solution to this problem is to extend the instrumentation on the side in question to a lower cervical level to achieve adequate bone purchase. This choice forces the surgeon to sacrifice motion segments for stability. Another, more novel, ap-

ABBREVIATIONS CCJ = craniocervical junction; $C V A=$ congenital vertebral anomaly; $O C=$ occipitocervical; $r$ BMP = recombinant bone morphogenetic protein; SED = spondyloepiphyseal dysplasia.

SUBMITTED December 1, 2014. ACCEPTED January 15, 2015.

INCLUDE WHEN CITING DOI: 10.3171/2015.1.FOCUS14787.

DISCLOSURE The authors report no conflict of interest concerning the materials or methods used in this study or the findings specified in this paper. 
proach is to place unilateral OC instrumentation on the side that is anatomically most favorable. This solution has been documented in the adult skull base literature after destabilizing far-lateral skull base procedures; ${ }^{1,10}$ however, we are unaware of any literature addressing this topic in pediatric patients with CVAs.

If hardware fails in a pediatric patient with a CVA after a bilateral Oc-C2 instrumentation and fusion, salvage choices are often limited. When considering the options, it is obvious that a unilateral OC construct has both advantages and disadvantages. The advantages include keeping the fusion to the minimum number of levels (usually Oc$\mathrm{C} 2$ ), avoiding hardware placement in potentially unsound locations, and placing screws in bone that is most favorable for instrumentation (the midline occipital keel and the C-2 pars or lamina). The disadvantages of unilateral OC fixation are the uncertainties regarding the biomechanical soundness of unilateral fixation and the absolute requirement that the patient wear a hard cervical orthosis at all times until the fusion matures. These concepts are further illustrated by our recent finding that children with congenital skeletal abnormalities are at increased risk for instrumentation-related failure. ${ }^{11}$ In evaluating these patients we now reason that, in certain circumstances, a secure up-front unilateral construct is better than "bilateral fixation at any cost." Diminutive or abnormal osseous anatomy may make the instrumentation placement challenging, but in our experience such an approach has been highly successful. In this paper we describe our experience using unilateral OC fixation constructs, in either a primary or a secondary procedure, in patients with skeletal abnormalities at the CCJ.

\section{Methods \\ Data Collection}

After receiving institutional review board approval, we conducted a retrospective review of all patients younger than 18 years who underwent surgical management of OC instability at our institution between 2001 and 2014. We identified a subset of those patients who underwent unilateral fixation of CCJ for management of OC instability, as either a primary or a secondary procedure. The cause of OC instability, reason for unilateral fixation, surgical details, and radiographic outcomes were recorded. To evaluate the presence or absence of fusion, all patients underwent a CT scan at least 4 months postoperatively.

The initial presence of OC instability was determined by identifying abnormal motion on flexion-extension radiographs and reviewing CT scans with 2D sagittal and coronal reconstructions. Previously described criteria were used to define the presence of OC subluxation, Oc$\mathrm{C} 1$ lateral displacement, or $\mathrm{Cl}-2$ lateral displacement. ${ }^{4}$

Bony fusion was defined as a solid bony bridge from the occiput to the posterior elements of C-2 on postoperative $\mathrm{CT}$ scans. The presence of partial bony bridging between the graft material and the occiput and $\mathrm{C}-2$, but without a clear gap between the graft-bone interfaces, was considered a fibrous union. Nonunion was defined by the resorption of graft material on subsequent postoperative CT scans.

\section{Surgical Technique}

All operations were performed by the senior author (D.L.B.). Details regarding surgical technique for OC fixation have been described previously. ${ }^{11}$ Briefly, our preference is to perform bilateral C-2 fixation by using pars screws with polyaxial heads. A rigid rod-plate device (Vertex, Medtronic Corp.) is used, with the rod secured into the top-loading C-2 screw with a locking cap and the plate anchored into the occipital bone with screws. The occipital bone and C-2 lamina are decorticated with a high-speed drill. Two ribs are harvested and secured to the occiput with maxillofacial screws. The rib grafts are held in place at the cervical end with a multistranded titanium cable (Atlas cable, Medtronic Corp.) wrapped around the rods. Demineralized bone matrix (DBX, Medtronic Corp.) is applied to the fusion mass. Recombinant bone morphogenetic protein (rBMP) (Infuse, Medtronic Corp.) is not routinely used. An intraoperative CT scan (O-Arm, Medtronic Corp.) is used to verify screw positioning at the end of the procedure. Postoperatively, patients are maintained in a custom rigid cervical orthosis until fusion has been documented.

For patients undergoing unilateral fixation, the following modifications are made. The occipital end of the rod-plate device is secured to the midline occipital keel rather than the thinner lateral regions of the occipital bone (Fig. 1). The C-2 screw is inserted into the pars or lamina on the chosen side. Pars screws are typically preferred to translaminar screws to maximize the bony surface of the posterior elements for fusion. The fusion construct may be augmented by sandwiching a small pledget of rBMP between the rib graft and native bone. Postoperatively, patients are maintained in a custom-built cervical orthosis at all times until a stable union is identified on CT scanning.

\section{Results}

We identified 8 patients who underwent unilateral fixa-

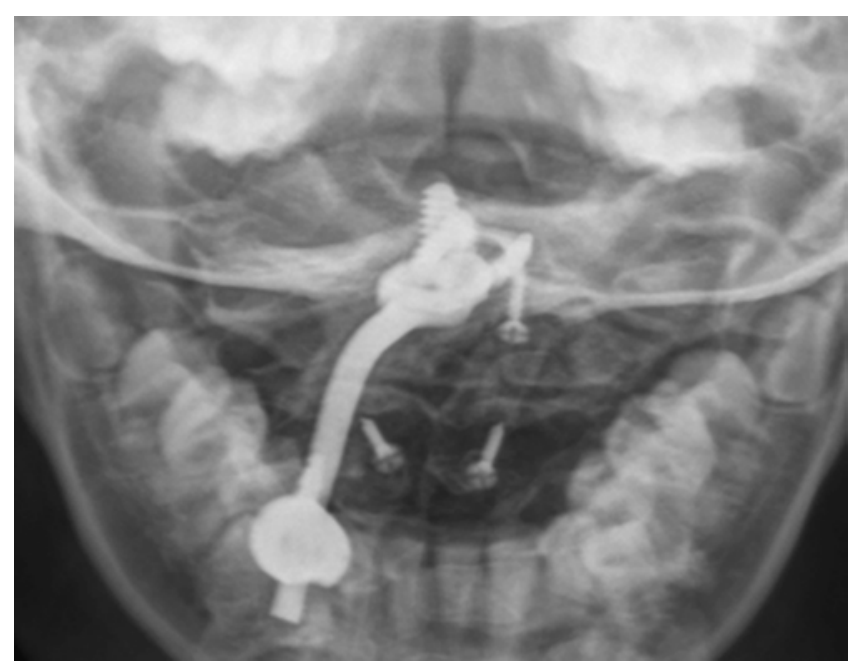

FIG. 1. Anteroposterior open-mouth odontoid radiograph demonstrating a unilateral rod-plate construct. The construct is secured at the cranial end with screws anchoring the plate in the midline occipital keel and at the cervical end with a nut locking the rod into the C-2 pars screw. Maxillofacial screws anchoring the rib autografts can also be seen. 
tion for management of OC instability. Patient characteristics, surgical details, and radiographic outcomes are described in Table 1. In all 8 patients a postoperative cervical spine CT scan was obtained at 4 months or later to evaluate osseous fusion. Each patient had CVAs of the CCJ: 2 patients had SED, 4 had atlantal hemi-rings, 1 had FG syndrome with dysplastic occipital condyles, and 1 had Down syndrome.

Unilateral fixation was performed during the initial procedure in 4 patients. In each patient, the contralateral side was determined to be unsuitable for screw insertion. In 3 patients, unilateral fixation was performed as a salvage procedure where, during the original procedure, fixation was obtained bilaterally but screw failure occurred on one side. The last patient, with OC instability due to a CVA and congenital C2-3 fusion, had bilateral C-3 fixation, but a wound infection required instrumentation and bone graft removal. After long-term antibiotic treatment, the patient underwent unilateral C-2 fixation.

Seven patients underwent C-2 pars screw placement at the time of unilateral fixation. One patient underwent C-2 translaminar screw placement, but this screw backed out and was replaced with a C-2 pars screw during the revision surgery. All patients had rib autografts. We used rBMP in 3 patients: 2 during the initial operation and 1 during a revision.

Five patients did not require revision surgery after the unilateral construct was placed, 2 patients required reoperation for loosening of the unilateral cervical screw, and 1 patient developed nonunion requiring revision of the bone graft but not the unilateral C-2 screw. Postoperative CT scans demonstrated osseous fusion in 7 patients and a fibrous union in 1 patient.

\section{Illustrative Cases}

\section{Case 1: Unilateral Instrumentation as an Initial Operation}

An infant was found incidentally to have multiple segmentation anomalies in the cervical spine during a workup for a tracheoesophageal fistula. Initial CT images revealed hypoplastic occipital condyles and an incomplete C-1 with poorly developed lateral masses (Fig. 2A). Gross OC instability with Oc-C1 lateral displacement was suspected, and the patient was placed in a custom-fitted rigid cervical orthosis. She wore this brace until she was 2 years of age, when her osseous structures were thought to have matured to the extent that $\mathrm{OC}$ instrumentation and fusion could be performed. A repeat CT scan showed that the occipital condyles remained hypoplastic; atlantal hemi-rings were also present.

A unilateral OC fusion was performed with a $3.5 \times 16-$ $\mathrm{mm}$ screw inserted into the right $\mathrm{C}-2$ pars (Fig. 2B); the left C-2 pars was too small for screw fixation (Fig. 2C). The C-2 screw was combined with a rod-plate device that was affixed to the midline occipital keel with $4.5 \times 6-\mathrm{mm}$ and $4.5 \times 8-\mathrm{mm}$ screws. Two rib autografts were harvested and secured at the occipital end with a $1.5 \times 8-\mathrm{mm}$ maxillofacial screw and at the cervical end with an Atlas cable. A small pledget of rBMP was inserted between the bone graft and the native bone on both the occipital and the cervical ends to augment the fusion. Postoperatively, the patient continued to wear her custom-fitted cervical orthosis. The CT images obtained at the 4-month followup demonstrated a solid osseous fusion, and the use of the orthosis was discontinued. Radiographs obtained at 6 months showed that the positions of the instrumentation and fusion construct were stable (Fig. 2D).

\section{Case 2: Unilateral Instrumentation as a Salvage Operation}

A 2-year-old girl with a short neck and developmental motor delay underwent surveillance imaging to evaluate for a possible genetic syndrome. Imaging demonstrated abnormal skeletal relationships at the CCJ, with the presence of 2 splayed atlantal hemi-rings that did not articulate with the lateral masses of C-2 (Fig. 3A and B). Severe OC instability was present, with brainstem compression.

An OC instrumentation and fusion operation was performed. A $3.5 \times 10-\mathrm{mm}$ screw was placed successfully in the left C-2 pars, but screw breakout occurred intraoperatively on the right, and instrumentation on this side was abandoned. Unilateral fixation was performed with the left $\mathrm{C}-2$ screw combined with a unilateral OC plate affixed to the occiput in the midline with $4 \times 10-\mathrm{mm}$ and $4 \times 6-\mathrm{mm}$ screws, respectively. Two rib autografts were harvested and secured with maxillofacial screws at the cranial and cervical ends and with a Songer cable. The patient was required to wear a custom-fitted cervical collar postoperatively.

One month later, routine postoperative radiographs demonstrated backout of the left C-2 pars screw (Fig. 3C). A revision operation was performed. The left C-2 screw had loosened and was replaced with a longer $3.5 \times 14-$ $\mathrm{mm}$ screw in the C-2 pars (Fig. 3D). Screw purchase was thought to be secure. A screw was inserted into the right C-2 lamina through a separate stab incision, but this screw had poor bone purchase and was abandoned. The unilateral OC plate was replaced with a rod-plate construct and affixed to the occiput by using $4 \times 6-\mathrm{mm}$ screws, and the C-2 screw was secured with a lock nut. The previous rib autografts were left in place because a fusion mass was present at the occipital end. Demineralized bone matrix was added to augment the cervical end of the fusion. The patient continued to wear her cervical orthosis postoperatively. The CT images obtained at 7 months demonstrated a successful fusion (Fig. 3E).

\section{Discussion}

This study demonstrates the utility of unilateral OC fixation for the treatment of children with CVAs of the CCJ. To our knowledge, this is the first report to describe the management of OC instability with the use of unilateral fixation during either a primary or a secondary procedure. Our results suggest that high fusion rates can be achieved using nonconventional biomechanical constructs. It is unclear whether it is the small-for-their-age nature of our patient population that makes unilateral fixation possible or whether these results can be generalized to other patient populations.

Previous authors have described the selective use of unilateral OC fixation in adults following a far-lateral approach that removes the occipital condyle and destabilizes 


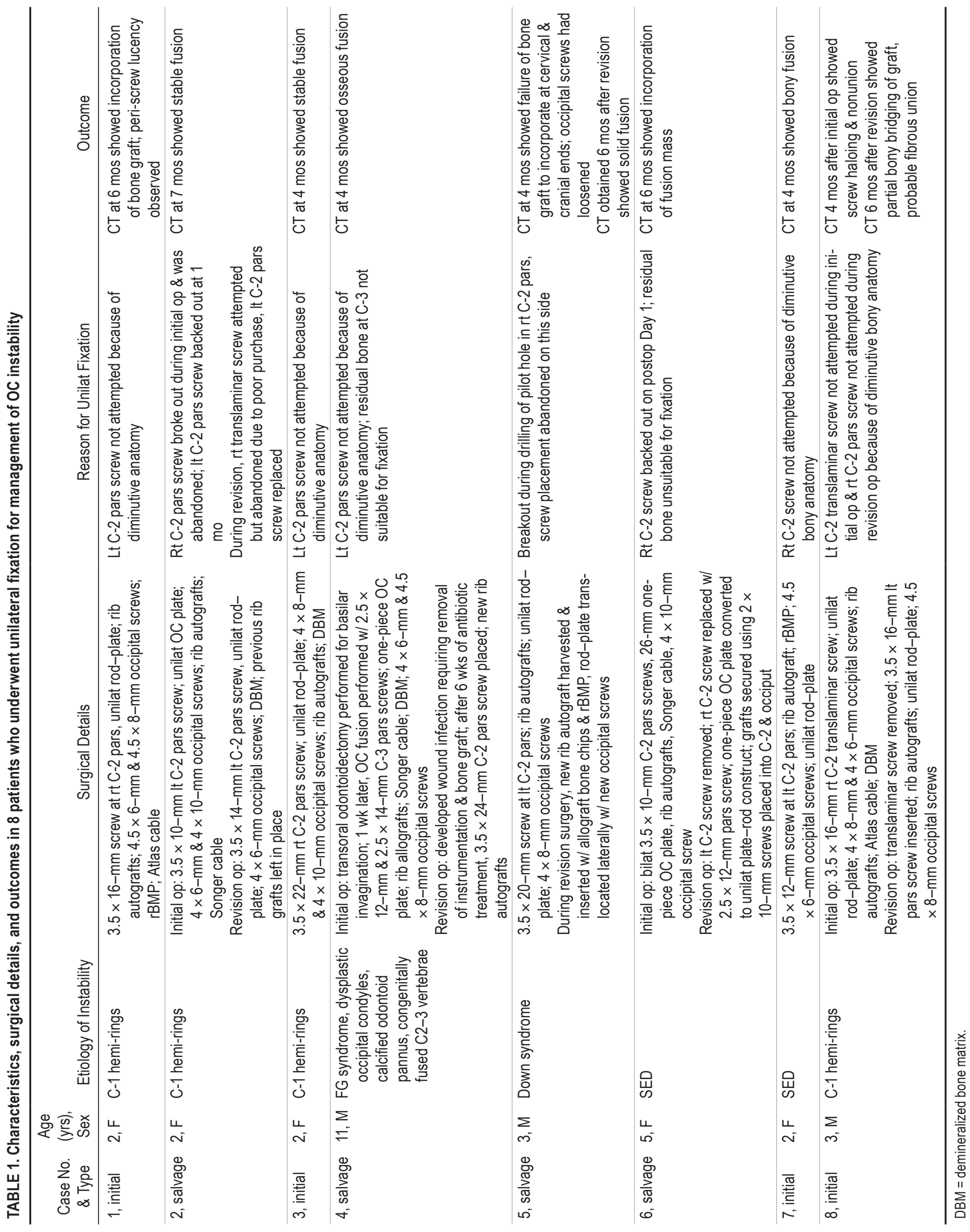



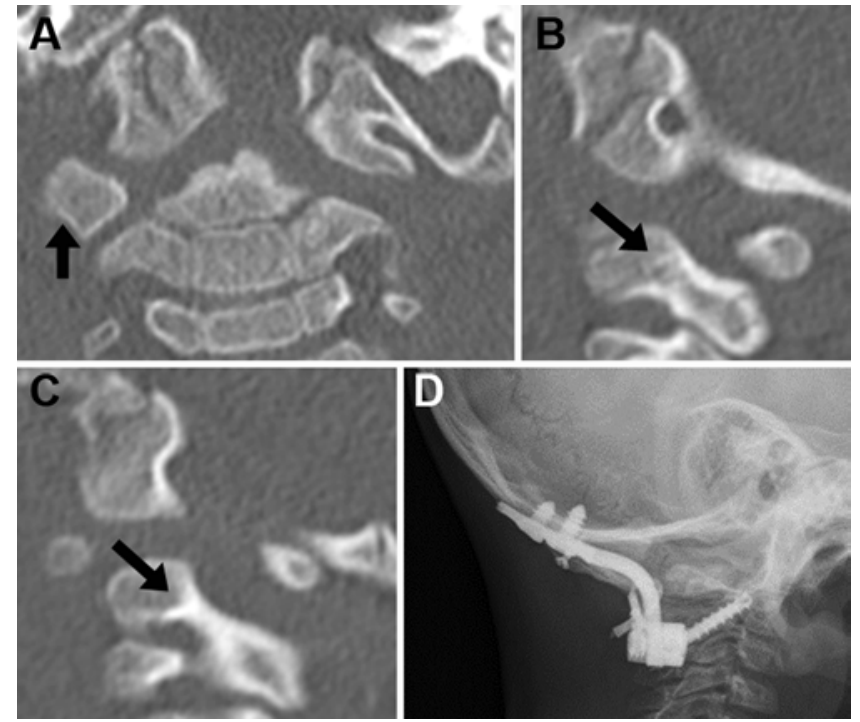

FIG. 2. A: Coronal CT scan demonstrating incomplete $\mathrm{C}-1$ arch with lateral displacement of $\mathrm{C}-1$ lateral mass (arrow) in relation to the occipital condyle. The hypoplastic left C-1 lateral mass is not seen in this plane. B: Sagittal CT scan showing right C-2 pars (arrow) that was amenable to screw fixation. C: Sagittal CT scan showing diminutive left C-2 pars (arrow) that was not suitable for screw fixation. D: Lateral radiograph obtained at 6 months demonstrating unilateral C-2 pars screw placement without complication.

the OC joint. ${ }^{1}$ Unilateral fixation performed using a single C1-2 transarticular screw has also been used to treat patients with atlantoaxial instability. ${ }^{3,4,6,8,11,12,15,16}$ Aside from these examples, we are unaware of any literature discussing the merits of unilateral instrumentation in OC instability. Other techniques described include in situ fusion, without rigid instrumentation, and wire fixation to the suboccipital bone..$^{5,7}$ Occipitocervical wiring has demonstrated acceptable fusion rates but is associated with high rates of complications, such as extension of fusion to unintended levels, CSF leaks, permanent neurological injury, and death. ${ }^{7}$ Both of these techniques commonly require prolonged external halo immobilization, which carries its own risks of pin-site and vest-related complications. ${ }^{5}$

In the setting of CVAs, structural abnormalities of the occipital condyles, C-1, or C-2 may cause OC instability. A wide variety of destabilizing CVAs has been described in the literature, including occipitalization of C-1, flattened occipitoatlantal joints, hypoplastic dens, and incomplete C-1 rings. ${ }^{4,8}$ Patients with skeletal dysplasia with or without congenital spinal anomalies may have malformed and diminutive osseous anatomy, making OC fusion very challenging. Furthermore, children with CVAs have a higher chance of instrumentation or fusion failure compared with children with normal anatomy. ${ }^{11}$ It is reasonable to ask why unilateral OC constructs might work in a patient population that is notorious for small anatomy, poor bone quality, and gross instability. One explanation might be that because of the smaller biomechanical forces created by young patients, unilateral constructs provided just enough stability to allow the arthrodesis to mature. It is a real-life demonstration of the old dictum, "successful arthrodesis is a race between the hardware failing and the fusion taking."

There are drawbacks to unilateral fixation. As we pointed out, unilateral constructs are biomechanically weak. The load of the cranium is distributed over fewer fixation points, which decreases stiffness against torsional forces when compared with bilateral constructs. The CCJ is highly mobile, and a unilateral construct does not robustly resist motion in rotation, lateral bending, or flexion-extension. The potential pitfalls of implanting the construct include not securing the cervical screw well enough, so that its pullout strength is compromised, or not securing the bone graft securely against the recipient fusion surface.

On the other hand, unilateral OC constructs may confer certain advantages. Unilateral instrumentation allows for more osseous surface area to be available for bony fusion than bilateral fixation, which is particularly relevant in young children with diminutive spines. Bilateral fixation may block the precious bony surface area, thus reducing the chance for success. Also, at the cranial end of
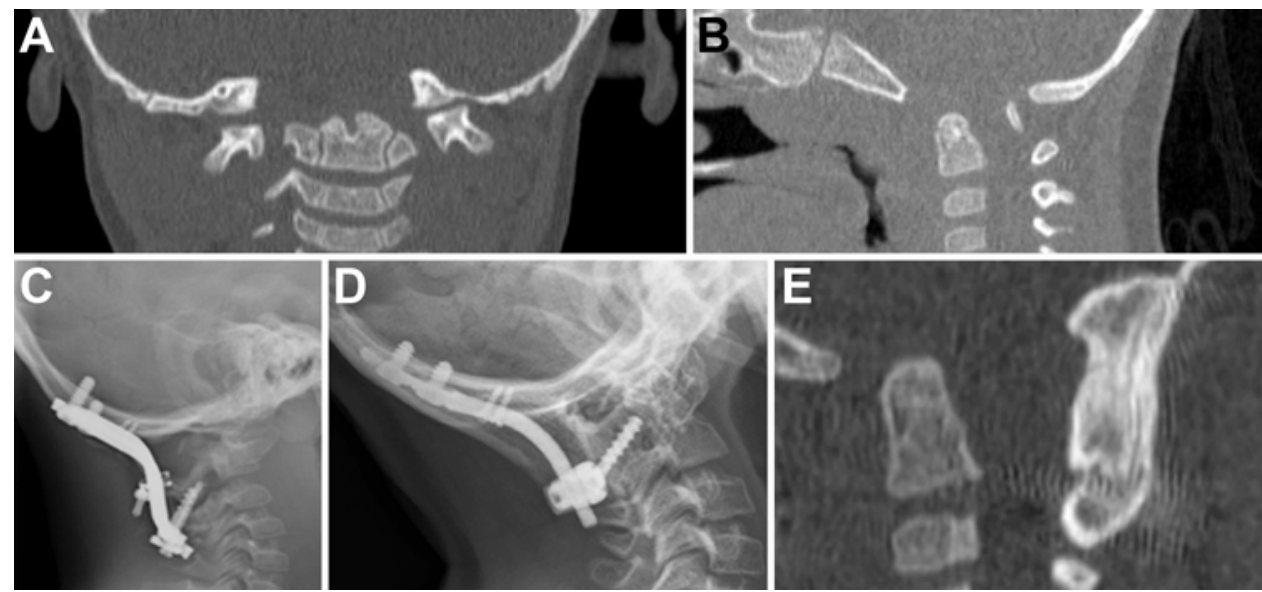

FIG. 3. A: Coronal CT scan demonstrating laterally displaced C-1 lateral masses in a patient with C-1 hemi-rings. B: Sagittal CT scan showing that the anterior $\mathrm{C}-1$ arch is incomplete and the odontoid is hypoplastic. The posterior $\mathrm{C}-1$ arch is causing severe stenosis. C: Lateral radiograph obtained at 1 month showing C-2 screw backout. D: The unilateral left C-2 pars screw was replaced; right C-2 screw insertion was attempted but abandoned because of poor purchase. E: Follow-up CT scan obtained at 7 months demonstrating a stable osseous fusion. 
the construct, screws are inserted in the midline occipital keel, thus maximizing their pullout strength. Furthermore, a unilateral construct can almost always keep the fusion level from occiput to $\mathrm{C}-2$, thus minimizing the number of motion segments required to achieve OC stability and preventing overly long fusion constructs.

On a technical note, rib autografts are used because they contain a lordotic curve and can be cut to fit the space between the occiput and C-2. The grafts are secured at the cranial end with maxillofacial screws and at the cervical end with a multistranded titanium cable. In some cases, we have chosen to use rBMP to augment the fusion by using it in sandwich fashion between the graft and the recipient site; however, we do not routinely use rBMP because of its potential side effects, such as spinal cord compression from a large postoperative seroma or heterotopic bone formation. ${ }^{9}$

Last, it is important to point out that OC motion must be restricted during the postoperative period to enable the osseous fusion to develop. This is accomplished by keeping patients in a rigid custom-fitted cervical collar at all times until fusion is identified on CT scans. Although a rigid cervical collar does not restrict motion to the same extent as a halo vest, a collar is much more easily tolerated and, as our results demonstrate, successful fusion can be obtained with its use.

This study is limited by its short follow-up (4-7 months). Previous studies have shown that osseous OC fusion can be seen on CT scans at 3 or 4 months after surgery, ${ }^{11,17}$ but the long-term outcomes of unilateral spinal instrumentation in a skeletally immature child are not yet known. A review of 17 children younger than 6 years who underwent $\mathrm{OC}$ and atlantoaxial fusions at our institution with a mean follow-up of 28 months demonstrated no evidence of new craniocervical deformity, subaxial instability, or unintended fusion of adjacent levels. ${ }^{2}$ Longer follow-up periods are needed to determine how children's spines mature after OC fusion.

\section{Conclusions}

Unilateral OC fixation may be suitable for the treatment of OC instability in children with CVAs of the CCJ in whom bilateral instrumentation placement fails or is not feasible. Only further use of this technique will ultimately determine whether it may become a viable up-front treatment option for patients with severe OC instability due to CVAs.

\section{References}

1. al-Mefty O, Borba LA, Aoki N, Angtuaco E, Pait TG: The transcondylar approach to extradural nonneoplastic lesions of the craniovertebral junction. J Neurosurg 84:1-6, 1996

2. Anderson RCE, Kan P, Gluf WM, Brockmeyer DL: Longterm maintenance of cervical alignment after occipitocervical and atlantoaxial screw fixation in young children. J Neurosurg 105 (1 Suppl):55-61, 2006

3. Brockmeyer D: Congenital vertebral anomalies, in Albright AL, Pollack IF, Adelson PD (eds): Principles and Practice of Pediatric Neurosurgery, ed 3. New York: Thieme, 2014, pp 364-380
4. Brockmeyer DL, Brockmeyer MM, Bragg T: Atlantal hemirings and craniocervical instability: identification, clinical characteristics, and management. J Neurosurg Pediatr 8:357-362, 2011

5. Elia M, Mazzara JT, Fielding JW: Onlay technique for occipitocervical fusion. Clin Orthop Relat Res (280):170-174, 1992

6. Gluf WM, Brockmeyer DL: Atlantoaxial transarticular screw fixation: a review of surgical indications, fusion rate, complications, and lessons learned in 67 pediatric patients. J Neurosurg Spine 2:164-169, 2005

7. Hwang SW, Gressot LV, Rangel-Castilla L, Whitehead WE, Curry DJ, Bollo RJ, et al: Outcomes of instrumented fusion in the pediatric cervical spine. J Neurosurg Spine 17:397409, 2012

8. Klimo P Jr, Rao G, Brockmeyer D: Congenital anomalies of the cervical spine. Neurosurg Clin N Am 18:463-478, 2007

9. Lindley TE, Dahdaleh NS, Menezes AH, Abode-Iyamah KO: Complications associated with recombinant human bone morphogenetic protein use in pediatric craniocervical arthrodesis. J Neurosurg Pediatr 7:468-474, 2011

10. Mazur MD, Brodke DS, Dailey AT: Restabilization of the occipitocervical junction after a complete unilateral condylectomy: a biomechanical comparison of unilateral and bilateral fixation techniques. Presented at the 42nd Annual Meeting of the Cervical Spine Research Society, Orlando, Florida, 2014 (Poster) (mosbrookdesign.com/CSRS/files/ EPoster_18.pdf) [Accessed February 15, 2015]

11. Mazur MD, Sivakumar W, Riva-Cambrin J, Jones J, Brockmeyer DL: Avoiding early complications and reoperation during occipitocervical fusion in pediatric patients. J Neurosurg Pediatr 14:465-475, 2014

12. Menezes AH: Craniocervical developmental anatomy and its implications. Childs Nerv Syst 24:1109-1122, 2008

13. Nichols LA, Mukherjee DP, Ogden AL, Sadasivan KK, Albright JA: A biomechanical study of unilateral posterior atlantoaxial transarticular screw fixation. J Long Term Eff Med Implants 15:33-38, 2005

14. Papagelopoulos PJ, Currier BL, Hokari Y, Neale PG, Zhao $\mathrm{C}$, Berglund LJ, et al: Biomechanical comparison of C1-C2 posterior arthrodesis techniques. Spine (Phila Pa 1976) 32:E363-E370, 2007

15. Song D, Maher CO: Spinal disorders associated with skeletal dysplasias and syndromes. Neurosurg Clin N Am 18:499514, 2007

16. Song GS, Theodore N, Dickman CA, Sonntag VKH: Unilateral posterior atlantoaxial transarticular screw fixation. J Neurosurg 87:851-855, 1997

17. Winegar CD, Lawrence JP, Friel BC, Fernandez C, Hong J, Maltenfort M, et al: A systematic review of occipital cervical fusion: techniques and outcomes. J Neurosurg Spine 13:5-16, 2010

\section{Author Contributions}

Conception and design: Brockmeyer, Mazur. Acquisition of data: all authors. Analysis and interpretation of data: all authors. Drafting the article: all authors. Critically revising the article: Brockmeyer. Reviewed submitted version of manuscript: Brockmeyer, Mazur. Approved the final version of the manuscript on behalf of all authors: Brockmeyer. Study supervision: Brockmeyer.

\section{Correspondence}

Douglas L. Brockmeyer, Division of Pediatric Neurosurgery, Primary Children's Hospital, University of Utah, 175 N. Medical Dr. E, Salt Lake City, UT 84132. email: neuropub@hsc.utah.edu. 